\title{
a Hyperpolygon?
}

\section{Steven Rayan and Laura P. Schaposnik}

Part of the spectacular success of the study of quiver representations is the realization that one may construct many interesting geometries-both old and new-from just the data of a directed graph. For us, a quiver will simply be a directed graph with nodes labelled by natural numbers and multiedges permitted. Familiar geometries such as those of projective space and Grassmannians can be constructed from a relatively simple graph, consisting of just two nodes and an arrow from one to the other, which is a so-called $A_{2}$ quiver. By increasing the complexity of the quiver, one can produce more interesting spaces.

Quivers and flag varieties. As suggested by the connection of projective space and Grassmannians to $A$-type quivers, the ADE Dynkin-type quivers play a fundamental role in the theory and capture many of its connections to geometry, representation theory, combinatorics, and physics.

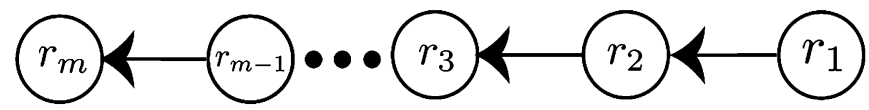

Figure 1. Labelled $A_{m}$ quiver.

Given an equioriented $A$-type quiver of length $m$ labelled by an increasing sequence of nonnegative integers $r_{i}$ as in Figure 1, one may associate the vector space

$$
V=\bigoplus_{i=1}^{m-1} \operatorname{Hom}\left(\mathbb{C}^{r_{i}}, \mathbb{C}^{r_{i}+1}\right)
$$

Steven Rayan is an associate professor of mathematics at the University of Saskatchewan. His email address is rayan@math. usask.ca.

Laura P. Schaposnik is an associate professor of mathematics at the University of Illinois at Chicago. Her email address is schapos@uic. edu.

Communicated by Notices Associate Editor Chikako Mese.

For permission to reprint this article, please contact:

reprint-permission@ams.org.

DOI: https://doi.org/10.1090/noti2204 which is subject to the conjugation action of the group $G=\prod_{i=1}^{m-1} \mathrm{GL}\left(r_{i}, \mathbb{C}\right)$. Through a suitable notion of quotient furnished either by geometric invariant theory (GIT) or by symplectic reduction, one may restrict to a subvariety of $V$ on which the group action is free. The result of restricting in this way and then quotienting is typically denoted by $V / / G$. The quiver variety $V / / G$ is an example of a moduli space, a space that keeps track of representations of the original quiver up to the equivalence furnished by $G$.

In the case of the $A$-type quiver, the quotient $V / / G$ is a partial flag variety $\mathcal{F}_{r_{1}, \ldots, r_{m}}$, from which the Grassmannians are recovered as $\mathcal{F}_{r_{1}, r_{2}}$. In the case of projective space $\mathbb{P}^{r-1}=\mathcal{F}_{1, r}$, GIT issues the familiar instruction of deleting the origin from $\operatorname{Hom}\left(\mathbb{C}, \mathbb{C}^{r}\right)$ before taking the quotient. For economy, we will denote the tuple of labels by $\underline{r}=\left(r_{1}, \ldots, r_{m}\right)$.

The partial flag varieties are prototypical examples of quiver varieties. One proceeds in essentially the same way for all quivers: an arrow between vertices labelled $u$ and $v$ contributes a summand $\operatorname{Hom}\left(\mathbb{C}^{u}, \mathbb{C}^{v}\right)$ to $V$ and there is a corresponding conjugation action by a product of general linear groups, whose factors act on the left or right of arrows via multiplication. Working over the field of complex numbers is useful for the correspondences we wish to motivate in this note, but one can choose to work over other fields.

Nakajima quiver varieties. An upgrade of the theory of quiver representations is provided by Nakajima, who considered the quaternionic vector space $T^{*} V$ rather than $V$ as the starting point for the quotient. Visually, for each existing arrow one adds another arrow that points in the opposite direction. The Nakajima quiver variety construction is symplectic in nature and involves restricting $T^{*} V$ to the intersection of two level sets, one given by the fiber of a moment map valued in the Lie algebra of a maximal 

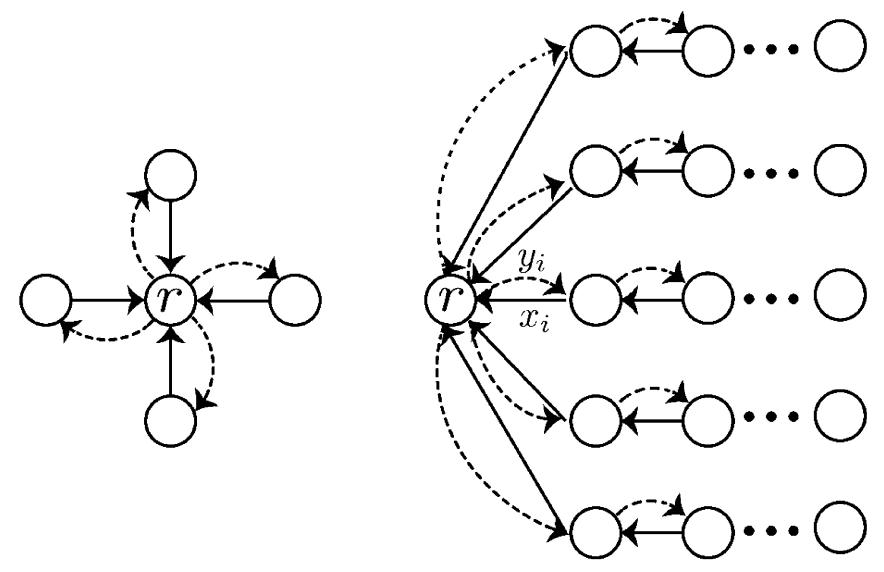

(a)

(b)

Figure 2. (a): Affine $D_{4}$ Dynkin diagram; (b): general

star-shaped quiver.

compact group in $G$ and the other in the Lie algebra of $G$ itself.

This quotient by $G$ is typically denoted $T^{*} V / / / G$. By the theorem of Hitchin-Karlhede-Linström-Roček, this quotient inherits three quaternionically-commuting complex structures $I, J, K$ from $T^{*} V$ as well as a Riemannian metric $g$. Antisymmetrizing $g$ by composing it with each of $I, J, K$ produces respective symplectic forms, $\omega_{I}, \omega_{J}, \omega_{K}$, giving three Kähler structures intertwined by $g$. This data on $T^{*} / / G$ is called a hyperkähler structure. Hyperkähler varieties hold a special place in geometry: for instance, they are automatically Calabi-Yau. For the case of the $A$-type quiver, the hyperkähler variety $T^{*} V / / G$ is precisely $T^{*} \mathcal{F}_{\underline{r}}$.

When the quiver is of any affine ADE type and is labelled appropriately, the corresponding Nakajima quiver variety is a gravitational instanton, in the sense that it is 2dimensional over $\mathbb{C}$ and has a complete, asymptotically locally Euclidean (ALE) metric $g$. The ALE condition means that the hyperkähler metric collapses to the Euclidean metric with polynomial order 4 as one goes out to infinity in any direction. In fact, every such gravitational instanton is realizable as a Nakajima quiver variety, completing a circle of ideas that includes the McKay correspondence and the work of Kronheimer.

Hyperpolygons. The story of hyperpolygons begins by considering a particular quiver, the affine $D_{4}$ Dynkin diagram, and generalizing it to a class of diagrams known as the starshaped quivers. The affine $D_{4}$ itself can be thought of as the interlacing of four copies of the $A_{2}$ quiver, as seen in Figure 2(a).

For any individual $A$-type quiver, allowing the copy of $\mathrm{GL}$ at the node with the largest label to act leads to a zerodimensional variety as the quotient. By interlacing $n A$ type quivers, each with length $m+1$ and labelled by a tuple $r^{i}=\left(r_{1}^{i}, \ldots, r_{m}^{i}, r\right)$ such that there is a common central node labelled by $r$, one obtains a star-shaped quiver. This is shown in Figure 2(b). The $A$-type quivers are referred to as the arms of the star. For $n$ sufficiently large, we can allow the group associated to the central node to act and still have a positive-dimensional quotient.

In order to build the Nakajima quiver variety for the star, we can use the fact that each $A$-type arm gives rise to $T^{*} \mathcal{F}_{r^{i}}$. The overall Nakajima quiver variety is the quotient of a subvariety inside the product of these cotangent bundles. This is an example of reduction in stages. From this point forward, we will adopt the symplectic point of view, so that the subvariety is an intersection of level sets of moment maps, and we shall restrict the action to that of the compact group $G=\mathrm{SU}(r)$. This subvariety is the set of simultaneous solutions to the (rescaled) moment map equations

$$
\begin{aligned}
\sum_{i=1}^{n}\left(x_{i} x_{i}^{*}-y_{i}^{*} y_{i}\right)_{0} & =0, \\
\sum_{i=1}^{n}\left(x_{i} y_{i}\right)_{0} & =0
\end{aligned}
$$

for $x_{i} \in \operatorname{Hom}\left(\mathbb{C}^{r_{m}^{i}}, \mathbb{C}^{r}\right)$ and $y_{i} \in T_{x_{i}}^{*} \operatorname{Hom}\left(\mathbb{C}^{r_{m}^{i}}, \mathbb{C}^{r}\right) \cong$ $\operatorname{Hom}\left(\mathbb{C}^{r}, \mathbb{C}^{r_{m}^{i}}\right)$, and where the subscript 0 is an instruction to make the matrix traceless by subtracting a multiple of the identity. One of the featured connections of these quotients to geometric representation theory is hidden in the left-hand side of equation (2). When we have just a single $A$-type arm, the image of this map is contained in the nilpotent cone of the dual of the Lie algebra $\mathfrak{g} \mathfrak{l}(r, \mathbb{C})$. When the flag is complete, $(1,2, \ldots, r)$, the map coincides with the famous Springer resolution.

A hyperpolygon is nothing more than an isomorphism class $\left[\left(x_{1}, \ldots, x_{n} ; y_{1}, \ldots, y_{n}\right)\right]$ in $T^{*} V / / / G$ for the star-shaped quiver. As a solution to the equations above, the data $x_{1}, \ldots, x_{n} ; y_{1}, \ldots, y_{n}$ defines a pair of polygons, one in the (rescaled) Lie algebra $\mathfrak{s} \mathfrak{u}(r)^{*}$ and another in $\mathfrak{g l}(r, \mathbb{C})^{*}$, with sides given by $\left(x_{i} x_{i}^{*}-y_{i}^{*} y_{i}\right)_{0}$ in the former case and by $\left(x_{i} y_{i}\right)_{0}$ in the latter. For the case of $r=2$ and the ordinary quotient $V / / G$ where all of the $y_{i}$ 's are zero, we recover ordinary $n$-gons in Euclidean 3 -space.

It is worth noting that, when $T^{*} \mathcal{F}_{\underline{r}}$ was constructed for each arm, the corresponding quotient had involved its own choice of level sets of real and complex moment maps. When the smallest label on each arm is $r_{1}^{i}=1$, this level set is determined by a number $\alpha_{i} \in \mathbb{R}$, which determines the fiber of the moment map for the action of the circle group $\mathrm{U}(1)$ on the outermost node of each arm. (All other moment maps can be set to 0 .) The choice of this number determines the Kähler structure on the underlying flag variety of each arm and determines the length of the $i$ th side $\left(x_{i} x_{i}^{*}-y_{i}^{*} y_{i}\right)_{0}$ of the real polygon. We will proceed with the assumption of $r_{1}^{i}=1$ from now on. 


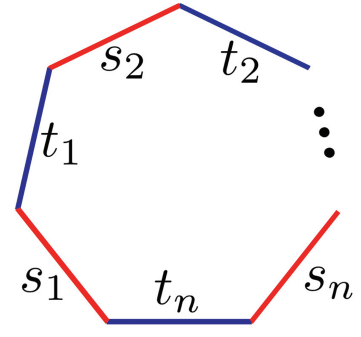

(a)

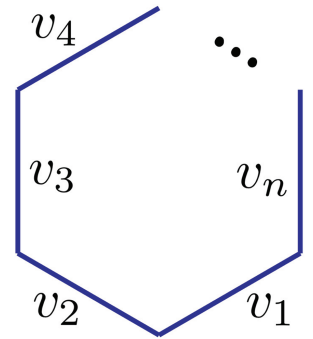

(b)
Figure 3. A hyperpolygon with $s_{i}=\left(x_{i} x_{i}^{*}\right)_{0}, t_{i}=-\left(y_{i} y_{i}^{*}\right)_{0}$, and $v_{i}=\left(x_{i} y_{i}\right)_{0}$.

Given this geometric interpretation, we refer to $T^{*} V / / / G$ for the star-shaped quiver as hyperpolygon space and give it its own symbol, $x_{r^{1}, \ldots, r^{n}}(\underline{\alpha})$, where $\underline{\alpha}$ is the vector of parameters $\alpha_{i} \in \mathbb{R}$. Hyperpolygon spaces were first studied by Konno as a hyperkähler extension to the notion of usual polygon space, and then later studied as a Nakajima quiver variety by Harada-Proudfoot. In a different vein, one can see in the moment map equation $\sum_{i=1}^{n}\left(x_{i} y_{i}\right)_{0}=0$ a connection to what is usually called the additive DeligneSimpson problem, which asks whether we can find representatives $C_{1}, \ldots, C_{n}$ of respective fixed conjugacy classes $\mathcal{C}_{1}, \ldots, \mathcal{C}_{n}$ in $\mathfrak{g l}(r, \mathbb{C})$ such that

$$
C_{1}+\cdots+C_{n}=0
$$

The conjugacy classes are fixed nilpotent orbits, and a solution to the general additive problem can be obtained by studying the problem from the point of view of starshaped quivers, as done by Crawley-Boevey.

Higgs bundles. Of particular interest is the connection of hyperpolygons to Higgs bundles, geometric objects on Riemann surfaces that arise from gauge theory as solutions to reduced self-dual Yang-Mills equations, now known as the Hitchin equations. Specifically, a Higgs bundle $(E, \phi)$ on a Riemann surface $X$ is a holomorphic vector bundle $E \rightarrow X$ with a global holomorphic map $\phi: E \rightarrow E \otimes \omega_{X}$, the Higgs field, where $\omega_{X}$ is the holomorphic cotangent bundle of $X$.

Higgs bundles of a fixed rank and Chern class come with their own moduli space, known as the Hitchin system. Like the Nakajima quiver varieties, the Hitchin system is hyperkähler. The term "system" refers to the fact that the moduli space of Higgs bundles contains the data of a completely integrable Hamiltonian system with respect to one of the symplectic forms of its hyperkähler structure. Another feature shared by Nakajima quiver varieties and the Hitchin system is an algebraic $\mathbb{C}^{*}$-action whose fixed-point locus contains all of their nontrivial cohomological information. In a sense, Nakajima quiver varieties are a finitedimensional analogue of the Hitchin system, which arises from the hyperkähler quotient of an infinite-dimensional affine space by a gauge group.

For the star-shaped quiver, the relationship between Nakajima quiver varieties and Hitchin systems is more than an analogy. The earliest work on this relationship is due to Godinho-Mandini, who considered flags of type $(1,2)$. More generally, choose any star-shaped quiver (with any flag types) and $n$ distinct points $z_{i} \neq \infty$ on the projective line $\mathbb{P}^{1}$. Then, setting

$$
\phi(z)=\sum_{i=1}^{n} \frac{\left(x_{i} y_{i}\right)_{0}}{z-z_{i}} d z,
$$

one obtains a meromorphic Higgs field, with simple poles at points $z_{i}$, for the trivial rank-r holomorphic bundle on $\mathbb{P}^{1}$. The moment map condition $\sum\left(x_{i} y_{i}\right)_{0}=0$ from equation (2) is a condition on the residues of this Higgs field that forces the Higgs field to be holomorphic at infinity.

For sufficiently generic choices of the Kähler moduli $\alpha_{i} \in \mathbb{R}$, the map sending a hyperpolygon $\left[\left(x_{1}, \ldots, x_{n} ; y_{1}, \ldots, y_{n}\right)\right]$ to the corresponding Higgs field $\phi(z)$ is a well-defined embedding of moduli spaces, where the target space is a moduli space of so-called parabolic Higgs bundles on a copy of $\mathbb{P}^{1}$ punctured along the divisor of the $n$ points. The parabolic structure-in particular, the parabolic weights-of the target moduli space are determined by the $\alpha_{i}^{\prime}$ s.

The construction of the Higgs bundle moduli space requires the restriction to certain Higgs bundles, determined by GIT or by moment maps (the Hitchin equations themselves). It turns out that hyperpolygons for generic $\alpha_{i}{ }^{\prime}$ s give rise to Higgs bundles that satisfy this restriction. The embedding of $x_{\underline{r}^{1}, \ldots, r^{n}}(\underline{\alpha})$ into a corresponding Higgs bundle moduli space is not hyperkähler-that is, it respects the $I$ complex structures of the two moduli spaces, but not the $J$ and $K$ ones. It is worth noting that, by convention, the $J$ and $K$ complex structures on the Hitchin system give it the interpretation as a moduli space of flat connections or as a character variety rather than as a space of Higgs bundles.

As an example, consider the affine $D_{4}$ quiver with the flag $(1,2)$ on each arm. The hyperpolygon space $\left.x_{(1,2),(1,2),(1,2),(1,2)} \underline{\alpha}\right)$ is a noncompact surface of dimension 2 over $\mathbb{C}$ that admits the structure of a gravitational instanton, by the Kronheimer-McKay-Nakajima correspondence. As such, the Nakajima hyperkähler metric $g_{N}$ has the ALE decay rate mentioned above. The corresponding parabolic Hitchin system on $\mathbb{P}^{1}$ with four simple poles is also a 2-dimensional space over $\mathbb{C}$. Despite the similarities, there is a codimension 1 locus in the Hitchin system that is not present in the hyperpolygon space, and the Hitchin metric also has a slower decay rate.

Generalized hyperpolygons. Inspired by conjectures of Hausel-Letellier-Rodriguez-Villegas, the authors of this note have extended the notion of hyperpolygon to a more general class of quivers, the comet-shaped quivers of Figure 4 .

These are formed by adding $g$-many loops to the central node of a star-shaped quiver. The loops are then doubled as per the Nakajima convention. A hyperkähler quotient 


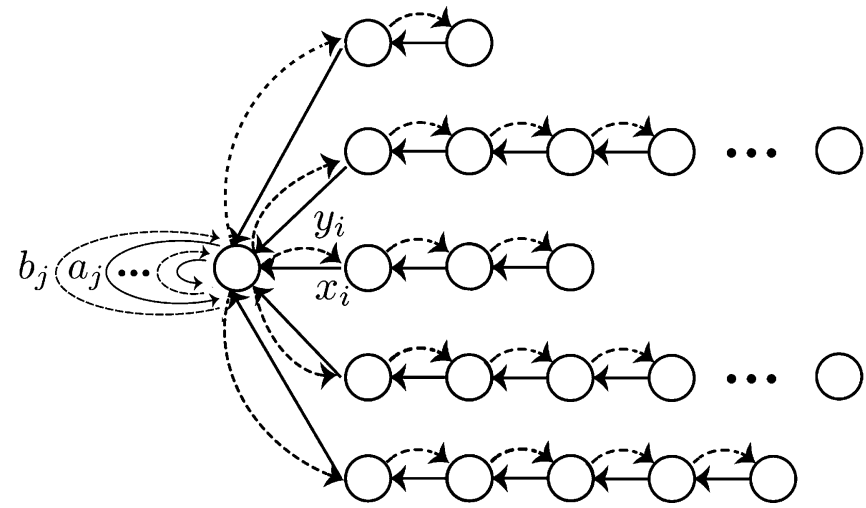

Figure 4. A comet-shaped quiver.

$T^{*} V / / / G$ is constructed as above for this quiver, with the resulting moduli space denoted as $X_{\underline{r}^{1}, \ldots, \underline{r}^{n}}^{g}(\underline{\alpha})$.

These generalized hyperpolygons can be interpreted again as a pair of polygons, but now with more sides due to the data representing the loops. The virtue of this generalization is that it also leads to a construction of an associated meromorphic Higgs bundle, but on a punctured Riemann surface of higher genus. In this setting, instead of puncturing the plane $\mathbb{C} \subset \mathbb{P}^{1} n$ times, one places either a regular parallelogram lattice on the plane or tessellates the hyperbolic plane by Poincaré regular $4 g$-gons. The fundamental cell is then punctured $n$ times in either scenario. The key then is to modify the denominator of the model Higgs field in equation (3) with an appropriate periodic function that maps each puncture to the cell in which our coordinate $z$ resides, in order for $\phi(z)$ to be well-defined on the quotient by the lattice or by the Fuchsian group of the tessellation.

Once compactified, one obtains a Riemann surface of genus $g$ equipped with a meromorphic Higgs field for the trivial rank- $r$ bundle. As in the previous setting, one has an embedding of moduli spaces in the $I$ complex structures. A key piece of data now is that the sum of the residues $\left(x_{i} y_{i}\right)_{0}$ is not zero but rather $\sum_{j=1}^{g}\left[a_{j}, b_{j}\right]$, where the $a_{j}$ 's are matrices in $\mathfrak{g} \mathfrak{I}(r, \mathbb{C})$ that represent the loops and where the $b_{j}$ 's represent the corresponding doubled loops as in Figure 4. This expression is the linearization of a product that defines the SL $(r, \mathbb{C})$-character variety of the Riemann surface, and speaks to the manner in which the hyperpolygon space collapses more rapidly to a Euclidean affine space at infinity than the Hitchin system.

Integrability. Since the space $X_{\underline{r}^{1}, \ldots, \underline{r}^{n}}^{g}(\underline{\alpha})$ is the quotient of

$$
T^{*} \mathcal{F}_{\underline{r}^{i}}\left(\alpha_{1}\right) \times \cdots \times T^{*} \mathcal{F}_{\underline{r}^{n}}\left(\alpha_{n}\right) \times T^{*} \mathfrak{g l}(r, \mathbb{C})
$$

by $\mathrm{SU}(r)$ subject to the appropriate moment map conditions, it inherits its symplectic structures from those of the hyperkähler varieties in the original product. These symplectic structures are host to integrable systems of GelfandTsetlin-type and, in the case of complete or minimal flags, can be written down explicitly in terms of Hamiltonians given by invariant polynomials of matrices.

The question is whether the integrable system descends along with the symplectic structures. In the case where every arm is either the minimal one $(1, r)$ or the complete one $(1,2, \ldots, r)$, then the answer is yes. This can be justified by working explicitly with the SU(r)-action and carefully tallying the number of such invariants that remain free after quotienting by the action. This number is exactly half the (complex) dimension of $x_{r^{1}, \ldots, r^{n}}^{g}(\underline{\alpha})$. What this establishes is the existence of explicit subintegrable systems within parabolic Hitchin systems. These subintegrable systems are defined using only representation-theoretic data and not the complex structure on the Riemann surface, which the Hitchin system relies upon in an intimate way. In a sense, these are very basic models for Hitchin integrability.

In general, through hyperpolygons we have a testing ground for various insights and conjectures into Hitchin systems. Hence, questions about Higgs bundles, such as explicit realizations of their mirror symmetry, can be asked about hyperpolygons with the potential for answers of a very concrete nature. Hyperpolygons emerge elsewhere in mathematics and physics, too, such as in quiver gauge theories and symplectic duality. Many themes in mathematics-even very deep and long-brewed oneshave at their heart examples that are very simple yet illuminating. Hyperpolygons play such a role in these corners of geometry, representation theory, and physics.

\section{Selected Further Reading}

Jonathan Fisher and Steven Rayan, Hyperpolygons and Hitchin systems, Int. Math. Res. Not. IMRN 2016, no. 6, 1839-1870. MR3509942

Laura Schaposnik, Higgs Bundles-Recent Applications, Notices Amer. Math. Soc. 67 (2020), no. 5, 625-634.

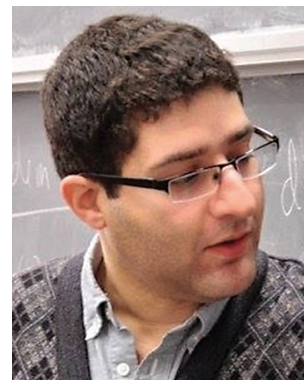

Steven Rayan

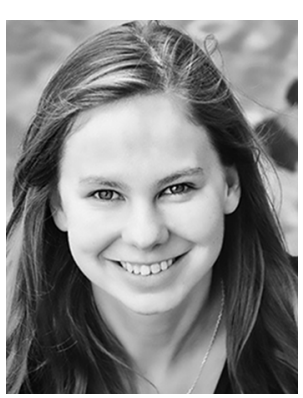

Laura P. Schaposnik

\section{Credits}

All figures are courtesy of the authors.

Photo of Steven Rayan is courtesy of Steven Rayan.

Photo of Laura P. Schaposnik is courtesy of Laura P. Schaposnik. 